\title{
Spotlight on tavaborole for the treatment of onychomycosis
}

This article was published in the following Dove Press journal:

Drug Design, Development and Therapy

20 November 2015

Number of times this article has been viewed

\section{Sphoorthi Jinna \\ Justin Finch}

Department of Dermatology, University of Connecticut School of Medicine, Farmington, CT, USA
Correspondence: Justin Finch

Department of Dermatology,

University of Connecticut School of

Medicine, 2I South Road, Farmington,

CT 06032, USA

Tel +l 8606794600

Fax +I 8606794759

Email finch@uchc.edu

\begin{abstract}
Onychomycosis is a fungal nail plate infection that has been increasing in prevalence. A variety of oral and topical anti-fungal agents are currently available but their use is limited by their adverse effect profile, drug-drug interactions, and limited efficacy. Therefore, there is a great need for newer anti-fungal agents. Tavaborole is one of these newer agents and was approved by the US Food and Drug Administration in July 2014 for the topical treatment of mild to moderate toenail onychomycosis. Tavaborole is a novel, boron-based anti-fungal agent with greater nail plate penetration than its predecessors, due to its smaller molecular weight. It has proven through several Phase II and III trials that it can be a safe and effective topical agent for the treatment of mild to moderate toenail onychomycosis without the need for debridement. In this paper, we review the landscape of topical and systemic treatment of onychomycosis, with particular attention to the pharmacokinetics, safety, and efficacy of topical tavaborole.
\end{abstract}

Keywords: tavaborole, boron-based antifungals, oxaboroles, onychomycosis

\section{Introduction}

Onychomycosis is a fungal nail plate infection affecting between $10 \%$ and $30 \%$ of the global population ${ }^{1,2}$ and accounting for approximately one-half of all nail disorders seen in dermatology practices. ${ }^{3}$ Prevalence of the disease continues to increase, partly due to the growing elderly population, ${ }^{4}$ the increasing number of patients with diabetes mellitus, ${ }^{5,6}$ and the increase in peripheral vascular disease. ${ }^{7}$ If left untreated, it can spread to adjacent nails, to additional body sites, or to other individuals. ${ }^{8}$ Goals of onychomycosis therapy therefore include mycological cure (negative potassium hydroxide $[\mathrm{KOH}]$ or fungal culture) and a normal appearing nail. Currently, a variety of anti-fungal options, both topical and oral, are available. ${ }^{9}$ While oral medications are the gold standard of onychomycosis therapy, the side effect profile and drug-drug interactions are limiting to many patients. ${ }^{10}$ Therefore, effective topical remedies are heavily sought after by both patients and providers. Successful treatment, however, is often hindered by the topical agents' limited ability to penetrate the nail. ${ }^{11}$ This can lead to a high incidence of relapse, often making onychomycosis a chronic condition for patients. ${ }^{12,13}$

\section{Current antifungal agents}

Before discussing the novel mechanism of the new class of boron-containing antifungals ushered in by tavaborole, it is worthwhile to review the existing antifungal landscape. Currently, there are several approved classes of antifungal drugs for the treatment of onychomycosis: allylamines, azoles, morpholines, and hydroxypyridinones, most of which target fungal cell membrane synthesis. ${ }^{14}$ 
Drugs of the allylamine class, including naftifine, butenafine, and terbinafine, inhibit squalene epoxidase, thereby blocking fungal ergosterol synthesis. ${ }^{15}$ Ergosterol is a sterol integral to fungal cell membrane synthesis, and without it many fungi cannot survive. Terbinafine $(250 \mathrm{mg}$ ) taken orally for 12 weeks is considered the current gold standard in onychomycosis therapy. ${ }^{16-18}$ Terbinafine's superior efficacy may be due to the fact that it is strongly lipophilic and therefore distributes well into skin, fat, and nails. ${ }^{16}$ While this therapy is the gold standard, mycologic cure rates with terbinafine are $73 \%-81 \%$, with complete cute rates merely $23 \%-38 \%$, indicating that current gold standards are still far from ideal. Also, periodic monitoring of liver function tests is recommended with terbinafine due to potential hepatotoxicity associated with this drug. ${ }^{16}$

The azole drug class also inhibits ergosterol biosynthesis, but does so via inhibition of lanosterol $14 \alpha$-demethylase. ${ }^{15}$ This class can be subdivided into imidazoles and triazoles based on the drugs' molecular structures. ${ }^{15}$ Currently, US Food and Drug Administration (FDA)-approved oral itraconazole ${ }^{19}$ and off-label oral fluconazole ${ }^{20}$ are used in the treatment of onychomycosis and are considered second and third line therapies, respectively. In June 2014, topical efinaconazole $10 \%$ nail solution was FDA approved for onychomycosis. ${ }^{21}$ Phase III trials indicated 15\%-18\% complete cure and $53 \%-56 \%$ mycologic cure with this topical regimen. This efinaconazole formulation represents an important advance in topical treatment of onychomycosis, but detailed discussion is beyond the scope of this paper.

The morpholine drug class includes topical amorolfine, which is approved in Europe but not in the US. ${ }^{22}$ Amorolfine works to deplete ergosterol as well, through inhibition of D14 reductase and D7-D8 isomerase. ${ }^{23}$ Ciclopirox is a hydroxypyridinone drug with a poorly understood mechanism of action that may involve iron chelation or oxidative damage. ${ }^{24,25}$

Finally, griseofulvin deserves mention as it was the first oral antifungal agent specifically approved for onychomycosis treatment in the US. ${ }^{26}$ A meta-analysis of three randomized trials using griseofulvin found a mycologic cure rate of approximately $60 \%$ with complete cure rates as low as $2 \%$. Compared with the other systemic agents, griseofulvin has little role in current anti-fungal therapy given these low efficacy rates, high relapse rates, long treatment durations (12-18 months), and drug interactions as a potent CYP3A4 inhibitor. ${ }^{26}$

\section{Topical antifungal therapy}

There has been a recent emergence of topical anti-fungal agents with improved nail permeation and efficacy.
Drug penetration through the nail plate is mostly predicted by low molecular weight in addition to the hydrophilic properties of the drug. ${ }^{27}$ Varying the molecular targets of these new therapies can be invaluable as well, especially considering the high proportion of incomplete onychomycosis treatment leading to antifungal resistance. ${ }^{28}$ Until June 2014, ciclopirox 8\% nail lacquer was the only topical agent approved for onychomycosis in North America. ${ }^{29,30}$ Two double-blind, vehiclecontrolled studies demonstrated complete cure (defined as clear nail and negative mycology by negative $\mathrm{KOH}$ and culture) in $5.5 \%$ and $8.5 \%$ of participants using ciclopirox solution. Negative mycology was noted in $29 \%$ and $36 \%$ of individuals. ${ }^{31}$

\section{Tavaborole}

The newest class of topical antifungal therapies is a novel drug class of boron-containing compounds called oxaboroles. ${ }^{32}$ Boron has uniquely been found to bind functional groups at target sites of particular enzymes, thereby rendering them inaccessible and impairing their function. The first molecule in this class of drugs to achieve FDA approval in July 2014 is tavaborole (formerly AN2690), which is formulated as a $5 \%$ topical solution for the treatment of toenail onychomycosis (Table 1). ${ }^{33}$

\section{Nail penetration}

Tavaborole's low molecular weight (approximately half of most antifungals, such as terbinafine and efinaconazole) permits optimal nail plate penetration, superior to that of existing topical antifungal medications. ${ }^{34}$ In a head-to-head study with ciclopirox, the amount of tavaborole $5 \%$ solution penetrating an ex vivo cadaver fingernail plate was 250 -fold higher than ciclopirox $8 \%$ solution after 14 days of daily application $\left(524.7 \mu \mathrm{g} / \mathrm{cm}^{2}\right.$ vs $13.0 \mu \mathrm{g} / \mathrm{cm}^{2}$, respectively). ${ }^{32,35}$ In vitro nail penetration studies also show that tavaborole is better

\section{Table I Tavaborole at a glance}

$\begin{array}{ll}\text { Structure } & \begin{array}{l}\text { 5-fluoro- I,3-dihydro- I-hydroxy-2, I- } \\ \text { benzoxaborole } \\ \text { Inhibits cytoplasmic leucyl-transfer RNA } \\ \text { synthetase, interfering with fungal protein } \\ \text { synthesis } \\ \text { Mechanism topical solution } \\ \text { Commercial availability }\end{array} \\ \text { FDA-approved use } & \text { Apply } \times 48 \text { weeks }\end{array}$

Abbreviation: FDA, US Food and Drug Administration. 
able to penetrate human nails compared with ciclopirox $8 \%$ and amorolfine $5 \%$ nail lacquers. ${ }^{35,36}$

\section{Mechanism of action}

Tavaborole possesses a unique mechanism of action making it highly specific against pathogenic fungi ${ }^{37}$ Unlike existing antifungals that inhibit ergosterol synthesis (terbinafine, itraconazole, fluconazole, amorolfine, and efinaconazole) or microbial metabolism (ciclopirox), tavaborole interferes with protein synthesis in fungal cells by acting on cytoplasmic aminoacyl-transfer RNA (tRNA) synthetases. ${ }^{34}$ Tavaborole specifically binds with leucyl-tRNA synthetase in the editing site, thus preventing the synthesis of leucine-charged tRNAs and ultimately suppressing fungal cell activity. ${ }^{34}$ Tavaborole has a greater than 1,000-fold higher affinity for fungal leucyltRNA synthetase compared with the human version. ${ }^{34,38,39}$ Therefore, human exposure is very low, providing a wide margin of safety. ${ }^{40}$

\section{Antifungal activity}

Tavaborole's unique mechanism of action is shown to be effective across a wide range of fungal organisms. In in vitro studies, tavaborole shows minimal inhibitory concentrations (MICs) of $1-8 \mu \mathrm{g} / \mathrm{mL}$ against the dermatophytes Trichophyton mentagrophytes and Trichophyton rubrum (the primary causative agents of onychomycosis) and of $\leq 0.5-4 \mu \mathrm{g} / \mathrm{mL}$ against the dermatophytes Trichophyton tonsurans $(2-4 \mu \mathrm{g} / \mathrm{mL})$, Epidermophyton floccosum $(\leq 0.5 \mu \mathrm{g} / \mathrm{mL})$, Microsporum audouinii $(2 \mu \mathrm{g} / \mathrm{mL})$, Microsporum canis $(2 \mu \mathrm{g} / \mathrm{mL})$, and Microsporum gypseum $(2 \mu \mathrm{g} / \mathrm{mL}) .{ }^{41}$ Specifically, both T. rubrum and T. mentagrophytes indicated an $\mathrm{MIC}_{50}$ of $4 \mu \mathrm{g} / \mathrm{mL}$ and an $\mathrm{MIC}_{90}$ of $8 \mu \mathrm{g} / \mathrm{mL} .{ }^{41}$ The presence of $5 \%$ powdered keratin has no effect on the microbiological activity of the drug against T. rubrum (MIC $2 \mu \mathrm{g} / \mathrm{mL}$ ). ${ }^{37,41}$ The drug is also active against the far less common nondermatophytic molds, such as Aspergillus fumigatus (MIC $0.25 \mu \mathrm{g} / \mathrm{mL}$ ) and Fusarium solani (MIC $\leq 0.5 \mu \mathrm{g} / \mathrm{mL}$ ), and yeast such as Candida albicans (MIC $1 \mu \mathrm{g} / \mathrm{mL}$ ). ${ }^{41}$ Two weeks after the completion of 28 days of treatment with tavaborole $7.5 \%$, nail plate concentrations above these MIC ranges are maintained; furthermore, therapeutic levels remain in the nail up to 3 months after treatment cessation, with drug levels corresponding to 161 times the $\mathrm{MIC}_{90}$ for T. rubrum. ${ }^{30}$

\section{Phase II trials}

Three Phase II clinical trials were conducted for tavaborole; two as open-label trials and one as a double-blind, doseranging, placebo-controlled trial. ${ }^{42}$ They all evaluated the treatment of distal and lateral subungual onychomycosis (DLSO; the most common form of onychomycosis) of at least one great toenail with $20 \%-60 \%$ nail plate involvement. ${ }^{42}$

The Phase II portion of a Phase I/II open-label study examined the efficacy of tavaborole $7.5 \%$ in 15 adults aged $18-65$ years. ${ }^{30}$ Participants had mycologically $(\mathrm{KOH})$ confirmed onychomycosis of at least one great toenail, a clinical diagnosis of onychomycosis in at least six additional toenails, $\geq 80 \%$ involvement of both great toenails, and a combined great toenail plate thickness of $>3 \mathrm{~mm}$. Participants applied tavaborole $7.5 \%$ to all ten toenails and surrounding skin once daily for 28 days. Twenty-five great toenails were evaluated for efficacy by fungal culture or $\mathrm{KOH}$. After only 2 weeks of treatment, $88 \%(22 / 25)$ of toenails treated with tavaborole demonstrated conversion from positive to negative culture. After 4 weeks of treatment, 100\% mycologic clearance was noted. ${ }^{30}$

A second, double-blind, vehicle-controlled, dose-ranging Phase II study was performed to investigate three concentrations of tavaborole compared with vehicle. ${ }^{30}$ A total of 187 patients with $\mathrm{KOH}$ - and culture-confirmed DLSO of the great toenail and 20\%-60\% involvement of the target nail were enrolled in the study. Participants applied vehicle $(n=63)$, tavaborole $2.5 \%(n=33)$, tavaborole $5 \%(n=31)$, or tavaborole $7.5 \%(n=60)$ daily for 3 months and then three times per week for the subsequent 3 months. The primary efficacy endpoint was negative culture and either $\geq 2 \mathrm{~mm}$ of clear nail growth or an Investigator Static Global Assessment (ISGA) of clear or almost clear growth at 6 months. All concentrations of tavaborole demonstrated a significantly greater therapeutic effect compared with vehicle after 6 months of treatment (clearance rates: 14\% for vehicle, 27\% for $2.5 \%$ tavaborole, $26 \%$ for $5 \%$ tavaborole, and $32 \%$ for $7.5 \%$ tavaborole). ${ }^{30}$

Another Phase II trial was composed of 89 individuals with $\mathrm{KOH}$ - and culture-confirmed DLSO of one great toenail and $20 \%-60 \%$ involvement of the target nail. ${ }^{30}$ Cohort 1 $(n=30)$ was treated with tavaborole $5 \%$ daily for 6 months, cohort $2(n=30)$ was treated with a $7.5 \%$ concentration daily for 6 months, and cohort $3(n=29)$ was treated with a 5\% concentration daily for 12 months. The primary efficacy endpoint was a negative culture and either $\geq 2 \mathrm{~mm}$ of clear nail growth or an ISGA of clear or almost clear growth at 6 months for cohorts 1 and 2, and at 12 months for cohort 3 . After 6 months of treatment, efficacy rates were $43 \%$ and $53 \%$ for cohorts 1 and 2, respectively. Following 12 months of treatment, the efficacy rate was $14 \%$ in cohort $3 .^{30}$

A final Phase II trial investigated $1 \%$ and 5\% concentrations in 60 participants with mycologically confirmed DLSO 
of the great toenail and 20\%-60\% involvement of the target nail. ${ }^{30}$ One group $(n=30)$ applied tavaborole $1 \%$ daily for 6 months and the other group $(n=30)$ applied tavaborole 5\% daily for the first 30 days and then three times per week for the following 5 months. The primary efficacy endpoint was negative culture and either $\geq 2 \mathrm{~mm}$ of clear nail growth or an ISGA of clear or almost clear growth at 6 months. After 6 months of treatment with tavaborole, $30 \%$ and $50 \%$ efficacy rates were achieved with $1 \%$ and $5 \%$ concentrations, respectively. ${ }^{30}$

\section{Phase III trials}

Two duplicate, multicenter, double-blind, randomized, vehicle-controlled Phase III trials were conducted for tavaborole 5\% nail solution (trial $1, \mathrm{n}=601$; trial 2 , $\mathrm{n}=593$ ). Adults 18 years and older with DLSO of at least one great toenail with $20 \%-60 \%$ nail plate involvement without dermatophytoma or lunula involvement were enrolled. Subjects were randomized $2: 1$ to applying either tavaborole 5\% solution or vehicle once daily for 48 weeks. The primary efficacy endpoint was complete cure of the target great toenail defined as negative mycology ( $\mathrm{KOH}$ and culture) and completely clear nail at week 52 . The complete cure rates for tavaborole were $6.5 \%$ and $9.1 \%(P=0.001$ and $<0.0001$, respectively), statistically superior to vehicle. Mycological cure rates were also significantly higher at $31.1 \%$ and $35.9 \%$ $(P<0.001)$. Overall, once daily application of tavaborole $5 \%$ is significantly more effective than vehicle in the treatment of toenail onychomycosis. ${ }^{43}$ Comparison to other antifungal agents makes it an attractive therapeutic option as well (Table 2). ${ }^{26,44}$

\section{Safety}

Boron-containing compounds have been known for many years to exhibit high toxicity in insects, enabling their use as pesticides. ${ }^{45}$ But, in general, toxicity in mammals has not

Table 2 Tavaborole compared to other oral and topical antifungal agents for the treatment of toenail onychomycosis

\begin{tabular}{|c|c|c|c|}
\hline & $\begin{array}{l}\text { Mycologic cure } \\
\text { rate }\end{array}$ & $\begin{array}{l}\text { Clinical cure } \\
\text { rate }\end{array}$ & $\begin{array}{l}\text { Complete } \\
\text { cure rate }\end{array}$ \\
\hline \multicolumn{4}{|l|}{ Oral } \\
\hline Griseofulvin & $60 \%$ & & \\
\hline Terbinafine & $76 \%$ & $66 \%$ & \\
\hline \multirow[t]{2}{*}{ Itraconazole } & $59 \%$ (continuous) & $70 \%$ (continuous) & \\
\hline & $63 \%$ (pulse) & $70 \%$ (pulse) & \\
\hline Fluconazole & $48 \%$ & & $15 \%-36 \%$ \\
\hline \multicolumn{4}{|l|}{ Topical } \\
\hline Efinaconazole $10 \%$ & $53 \%-56 \%$ & & $15 \%-18 \%$ \\
\hline Ciclopirox 8\% & $29 \%-36 \%$ & & $5 \%-9 \%$ \\
\hline Tavaborole 5\% & $30 \%-36 \%$ & $26 \%-28 \%$ & $6 \%-10 \%$ \\
\hline
\end{tabular}

been established. (In fact, boron itself may be an essential mineral in humans. ${ }^{46}$ ) That said, isolated reports have shown developmental or postnatal damage due to boron-containing compounds in rodents. ${ }^{47,48}$ Tavaborole, however, appears to have an excellent safety profile.

In preclinical safety studies, a concentration of $10 \mu \mathrm{M}$ of tavaborole did not inhibit the cytochrome $\mathrm{P} 450$ isoforms CYP1A2, CYP2C9, CYP2C19, CYP2D6, or CYP3A4. ${ }^{30}$ Plasma levels of tavaborole were below the limits of quantitation after topical application of $0.25 \mathrm{~mL}$ of $7.5 \%$ solution of tavaborole once daily for 28 days to the toenails of 15 subjects with onychomycosis. ${ }^{30}$ Another study of 24 subjects found that only a small fraction of the topically applied dose of tavaborole is absorbed systemically and that the drug does not accumulate after repeated application. ${ }^{37}$ Therefore, the risk for drug interactions with tavaborole is low. ${ }^{30}$ Further studies are needed, however, to confirm this finding in long-term use. In all, Phase II and III studies, none of the serious adverse events that occurred were deemed related to tavaborole, whereas the frequency of treatment-related adverse events were comparable to vehicle. The most common adverse events were application site exfoliation $(2.7 \% \mathrm{vs}$ $0.3 \%$ in the vehicle group), application site erythema $(1.6 \%$ vs $0 \%)$, and dermatitis $(1.3 \%$ vs $0 \%) .{ }^{30}$ They were generally mild to moderate and transient. Treatment discontinuation rates were low $(2.5 \%$ and $0.8 \%$ in the two Phase III trials $){ }^{37}$

The carcinogenic potential of tavaborole was investigated in a traditional 2-year carcinogenicity study in mice and rats. ${ }^{43}$ In mice, tavaborole was applied topically at concentrations of $0 \%, 5 \%$ (clinically relevant concentration), $10 \%$, and $15 \%$. It was also administered orally to the rat at $0,12.5,25$, and $50 \mathrm{mg} / \mathrm{kg} /$ day. There were no tavaborole-related neoplastic findings in either species. This study provides evidence that tavaborole is not carcinogenic in rodents and is therefore suitable for long-term use in humans. ${ }^{49}$ In a broader study, genetic toxicology studies were completed with four benzoxaboroles and one boronic acid ester and none of them demonstrated genetic toxicology liabilities, thus supporting the favorable safety profiles of tavaborole. ${ }^{50}$

\section{Conclusion}

Onychomycosis is a fungal infection that is steadily growing in prevalence and is in desperate need for highly effective and safe treatment options. Oral agents, while effective, come with a significant side effect profile and concern about drug-drug interactions. Therefore, good topical agents that can penetrate the nail plate while being safe and effective are paramount. Topical agents have historically had limited 
efficacy given the low penetration through the nail plate. This leads to frustration, noncompliance, and recurrence in onychomycosis patients.

The emergence of a new class of boron-containing drugs is exciting. The boron-containing molecule tavaborole offers a unique mechanism of action among antifungal agents, inhibiting leucyl-tRNA synthetase. Other boron-containing drugs under development for dermatologic disease inhibit phosphodiesterase-4, with potential applications for psoriasis and atopic dermatitis. ${ }^{33}$

Tavaborole has been shown to effectively treat mild to moderate onychomycosis caused by dermatophytes without the need for debridement. It effectively penetrates the nail plate given its significantly lower molecular weight compared to other agents. While its mycologic cure rate $(30 \%-36 \%)$ is lower than oral antifungal agents $(50 \%-76 \%)$, it offers an important alternative to available topical antifungal therapies.

Long-term studies need to be performed to assess both the full potential of the drug by providing more time for healthy regrowth of the nail and to assess recurrence rates. Head-tohead comparison studies of the efficacy of tavaborole vs efinaconazole and ciclopirox for the treatment of onychomycosis would be of value. Given tavaborole's unique mechanism of action, studies evaluating its use in combination with systemic antifungal therapy - for example, in preventing recurrence after systemic treatment - would be of interest as well.

\section{Disclosure}

The authors report no conflicts of interest in this work.

\section{References}

1. Ghannoum MA, Hajjeh RA, Scher R, et al. A large-scale North American study of fungal isolates from nails: the frequency of onychomycosis, fungal distribution, and antifungal susceptibility patterns. J Am Acad Dermatol. 2000;43:641-648.

2. Di Chiacchio N, Suarez MV, Madeira CL, Loureiro WR. An observational and descriptive study of the epidemiology of and therapeutic approach to onychomycosis in dermatology offices in Brazil. An Bras Dermatol. 2013;88(Suppl 1):3-11.

3. Scher RK, Coppa LM. Advances in the diagnosis and treatment of onychomycosis. Hosp Med. 1998;34:11-20.

4. Baran R. The nail in the elderly. Clin Dermatol. 2011;29(1):54-60.

5. Gupta AK, Konnikov N, MacDonald P, et al. Prevalence and epidemiology of toenail onychomycosis in diabetic subjects: a multicentre survey. Br J Dermatol. 1998;139(4):665-671.

6. Gupta AK, Humke S. The prevalence and management of onychomycosis in diabetic patients. Eur J Dermatol. 2000;10(5):379-384.

7. Gupta AK, Gupta MA, Summerbell RC, et al. The epidemiology of onychomycosis: possible role of smoking and peripheral arterial disease. J Eur Acad Dermatol Venereol. 2000;14(6):466-469.

8. Vander Straten MR, Hossain MA, Ghannoum MA. Cutaneous infections dermatophytosis, onychomycosis, and tinea versicolor. Infect Dis Clin North Am. 2003;17:87-112.
9. Gupta AK, Simpson FC. New therapeutic options for onychomycosis. Expert Opin Pharmacother. 2012;13(8):1131-1142.

10. Gupta AK, Ryder JE, Skinner AR. Treatment of onychomycosis: pros and cons of antifungal agents. J Cutan Med Surg. 2004;8:25-30.

11. Murdan S. Drug delivery to the nail following topical application. Int J Pharm. 2002;236:1-26.

12. Piraccini BM, Sisti A, Tosti A. Long-term follow-up of toenail onychomycosis caused by dermatophytes after successful treatment with systemic antifungal agents. J Am Acad Dermatol. 2010;62(3):411-414.

13. Scher RK, Baran R. Onychomycosis in clinical practice: factors contributing to recurrence. Br J Dermatol. 2003;149(Suppl 65):5-9.

14. Welsh O, Vera-Cabrera L, Welsh E. Onychomycosis. Clin Dermatol. 2010;28:151-159.

15. Gupta AK, Sauder DN, Shear NH. Antifungal agents: an overview. Part II. J Am Acad Dematol. 1994;30:911-933.

16. Lamisil (terbinafine hydrochloride) Tablets [Prescribing Information 2013]. Novartis Pharmaceuticals Corporation; East Hanover, NJ.

17. Gupta AK, Paquet M, Simpson F, et al. Terbinafine in the treatment of dermatophyte toenail onychomycosis: a meta-analysis of efficacy for continuous and intermittent regimens. J Eur Acad Dermatol Venereol. 2013;27:267-272.

18. Sigurgeirsson B, Billstein S, Rantanen T, et al. L. I. O. N. Study: efficacy and tolerability of continuous terbinafine (Lamisil) compared to intermittent itraconazole in the treatment of toenail onychomycosis. Lamisil vs itraconazole in onychomycosis. Br J Dermatol. 1999;141(Suppl 56): $5-14$.

19. Sporanox (Itraconazole) Capsules. Drugs@FDA: FDA Approved Drug Products. 2012.

20. Gupta AK, Drummond-Main C, Paquet M. Evidence-based optimal fluconazole dosing regimen for onychomycosis treatment. J Dermatol Treat. 2013;24:75-80.

21. Gupta AK, Simpson FC. New pharmacotherapy for the treatment of onychomycosis: an update. Expert Opin Pharmacother. 2015;16(2):227-236.

22. Baran R, Feuilhade M, Combernale P, et al. A randomized trial of amorolfine $5 \%$ solution nail lacquer combined with oral terbinafine compared with terbinafine alone in the treatment of dermatophytic toenail onychomycoses affecting the matrix region. Br J Dermatol. 2000;142: 1177-1183.

23. Polak A. Preclinical data and mode of action of amorolfine. Dermatology. 1992;184(Suppl 1):3-7.

24. Lee REB, Liu TT, Barker KS, et al. Genome-wide expression profiling of the response to ciclopirox olamine in Candida albicans. J Antimicrob Chemother. 2005;55:655-662.

25. Belenky P, Camacho D, Collins JJ. Fungicidal drugs induce a common oxidative-damage cellular death pathway. Cell Rep. 2013;3:350-358.

26. Finch JJ, Warshaw EM. Toenail onychomycosis: current and future treatment options. Dermatol Ther. 2007;20(1):31-46.

27. Kobayashi Y, Komatsu T, Sumi M, et al. In vitro permeation of several drugs through the human nail plate: relationship between physicochemical properties and nail permeability of drugs. Eur J Pharm Sci. 2004;21:471-477.

28. Balkis MM, Leidich SD, Mukherjee PK, Ghannoum MA. Mechanisms of fungal resistance: an overview. Drugs. 2002;62:1025-1040.

29. Del Rosso JQ. The role of topical antifungal therapy for onychomycosis and the emergence of newer agents. J Clin Aesthet Dermatol. 2014; 7(7):10-18.

30. Gupta AK, Daigle D, Abramovits W. Tavaborole 5\% solution for onychomycosis. Skinmed. 2015;13(1):55-58.

31. Penlac Nail Lacquer (Ciclopirox) Topical Solution PI. Dermik Laboratories, A Division of Aventis Pharmaceuticals Inc; Berwyn, PA.

32. Elewski BE, Tosti A. Tavaborole for the treatment of onychomycosis. Expert Opin Pharmacother. 2014;15(10):1439-1448.

33. Anacor Pharmaceuticals Inc. FDA approves Anacor Pharmaceuticals' KERYDINTM (tavaborole) topical solution, $5 \%$ for the treatment of onychomycosis of the toenails [Internet]. 2014.

34. Rock FL, Mao W, Yaremchuk A, et al. An antifungal agent inhibits an aminoacyl-tRNA synthetase by trapping tRNA in the editing site. Science. 2007;316(5832):1759-1761. 
35. Hui X, Baker SJ, Wester RC, et al. In vitro penetration of a novel oxaborole antifungal (AN2690) into the human nail plate. J Pharm Sci. 2007; 96(10):2622-2631.

36. Baker SJ, Sanders V, Hold KM, et al. In vitro nail penetration profile of AN2690, a novel broad-spectrum antifungal agent in development for the topical treatment of onychomycosis. J Pharm Sci. 2007;96(10): 2622-2631.

37. Markham A. Tavaborole: first global approval. Drugs. 2014;74(13): 1555-1558.

38. Barak O, Loo DS. AN-2690, a novel antifungal for the topical treatment of onychomycosis. Curr Opin Invest Drugs. 2007;8(8):662-668.

39. Alley MR, Baker SJ, Beutner KR, Plattner J. Recent progress on the topical therapy of onychomycosis. Expert Opin Invest Drugs. 2007;16(2): $157-167$.

40. Dosik J, Lastella P, Yavel R, et al. The pharmacokinetics of AN2690 after topical application in patients with onychomycosis. J Invest Dermatol. 2011;131:S47.

41. Sanders V, Baker SJ, Alley MRK, et al. Microbiological activity of AN2690, A new antifungal agent in development for the topical treatment of onychomycosis. Poster session presented at: the 64th Annual Meeting of the American Academy of Dermatology; March; 2006; San Francisco, CA.

42. Gupta AK, Simpson FC. Investigational drugs for onychomycosis. Expert Opin Invest Drugs. 2014;23(1):97-106.

43. Elewski BE, Aly R, Baldwin SL, et al. Efficacy and safety of tavaborole topical solution, $5 \%$, a novel boron-based antifungal agent, for the treatment of toenail onychomycosis: Results from 2 randomized phase-III studies. J Am Acad Dermatol. 2015. pii: S0190-9622(15)01512-1.
44. Gupta AK. Systemic Antifungal Agents. Comprehensive Dermatologic Drug Therapy. Edinburgh: Elsevier; 2013:98-120.

45. Soriano-Ursúa MA, Das BC, Trujillo-Ferrara JG. Boron-containing compounds: chemico-biological properties and expanding medicinal potential in prevention, diagnosis and therapy. Expert Opin Ther Pat. 2014; 24(5):485-500.

46. Mastromatteo E, Sullivan F. Summary: international symposium on the health effects of boron and its compounds. Environ Health Perspect. 1994; 102(Suppl 7):139-141.

47. Liu JY, Huang Y, Menting R, et al. A boron dipyrromethene-phtalocyanine pentad as an artificial photosynthetic model. Chem Commun. 2013;49: 2998-3000.

48. Heindel JJ, Price CJ, Schwetz BA. The developmental toxicity of boric acid in mice, rats, and rabbits. Environ Health Perspect. 1994;102: $107-112$.

49. Ciaravino V, Coronado D, Lanphear C, Shaikh I, Ruddock W, Chanda S. Tavaborole, a novel boron-containing small molecule for the topical treatment of onychomycosis, is noncarcinogenic in 2-year carcinogenicity studies. Int J Toxicol. 2014;33(5):419-427.

50. Del Rosso JQ, Plattner JJ. From the test tube to the treatment room. J Clin Aesthet Dermatol. 2014;7(2):13-21.
Drug Design, Development and Therapy

\section{Publish your work in this journal}

Drug Design, Development and Therapy is an international, peerreviewed open-access journal that spans the spectrum of drug design and development through to clinical applications. Clinical outcomes, patient safety, and programs for the development and effective, safe, and sustained use of medicines are a feature of the journal, which

\section{Dovepress}

has also been accepted for indexing on PubMed Central. The manuscript management system is completely online and includes a very quick and fair peer-review system, which is all easy to use. Visit http://www.dovepress.com/testimonials.php to read real quotes from published authors.

Submit your manuscript here: http://www.dovepress.com/drug-design-development-and-therapy-journal 